\title{
Dural Calcitonin Gene-Related Peptide Produces Female- Specific Responses in Rodent Migraine Models
}

\author{
Amanda Avona, ${ }^{1}$ Carolina Burgos-Vega, ${ }^{1}{ }^{\circledR}$ Michael D. Burton, ${ }^{1}$ Armen N. Akopian, ${ }^{2}$-Theodore J. Price, ${ }^{1}$ \\ and Gregory Dussor ${ }^{1}$ \\ ${ }^{1}$ School of Behavioral and Brain Sciences, Center for Advanced Pain Studies, University of Texas at Dallas, Richardson, Texas 75080, and ${ }^{2}$ Department of \\ Endodontics, University of Texas Health Science Center at San Antonio, San Antonio, Texas 78229
}

\begin{abstract}
Migraine is the second leading cause for disability worldwide and the most common neurological disorder. It is also three times more common in women; reasons for this sex difference are not known. Using preclinical behavioral models of migraine, we show that application of calcitonin gene-related peptide (CGRP) to the rat dura mater produces cutaneous periorbital hypersensitivity. Surprisingly, this response was observed only in females; dural CGRP at doses from $1 \mathrm{pg}$ to $3.8 \mu \mathrm{g}$ produce no responses in males. In females, dural CGRP causes priming to a pH 7.0 solution after animals recover from the initial CGRP-induced allodynia. Dural application of interleukin- 6 causes acute responses in males and females but only causes priming to subthreshold dural CGRP (0.1 pg) in females. Intracisternal application of BDNF also causes similar acute hypersensitivity responses in males and females but only priming to subthreshold dural CGRP $(0.1 \mathrm{pg})$ in females. Females were additionally primed to a subthreshold dose of the NO-donor sodium nitroprusside $(0.1 \mathrm{mg} / \mathrm{kg})$ following dural CGRP. Finally, the sexually dimorphic responses to dural CGRP were not specific to rats as similar female-specific hypersensitivity responses were seen in mice, where increased grimace responses were also observed. These data are the first to demonstrate that CGRP-induced headache-like behavioral responses at doses up to $3.8 \mu \mathrm{g}$ are female-specific both acutely and following central and peripheral priming. These data further implicate dural CGRP signaling in the pathophysiology of migraine and propose a model where dural CGRP-based mechanisms contribute to the sexual disparity of this female-biased disorder.
\end{abstract}

Key words: CGRP; gender; headache; meningeal innervation; migraine; pain

\section{Significance Statement}

Calcitonin gene-related peptide (CGRP) has long been implicated in the pathophysiology of migraine, and CGRP-based therapeutics are efficacious for the treatment of migraine in humans. However, the location of action for CGRP in migraine remains unclear. We show here that application of CGRP to the cranial meninges causes behavioral responses consistent with headache in preclinical rodent models. Surprisingly, however, these responses are only observed in females. Acute responses to meningeal CGRP are female-specific and sensitization to CGRP after two distinct stimuli are also female-specific. These data implicate the dura mater as a primary location of action for CGRP in migraine and suggest that female-specific mechanisms downstream of CGRP receptor activation contribute to the higher prevalence of migraine in women.

\section{Introduction}

Migraine is a complex neurological disorder that is characterized by throbbing head pain, increased sensitivity to light, sound, and

Received Feb. 13, 2019; revised March 19, 2019; accepted March 21, 2019.

Author contributions: A.A., C.B.-V., M.D.B., A.N.A., T.J.P., and G.D. designed research; A.A. and C.B.-V. performed research; A.A. and C.B.-V. analyzed data; A.A. wrote the first draft of the paper; A.A., M.D.B., and G.D. wrote the paper; M.D.B., A.N.A., T.J.P., and G.D. edited the paper.

This work was supported by National Institutes of Health Grant NS072204 to G.D. and Grant NS104200 to G.D. and A.A. We thank Nandita Ramkumar, Marina Motina, and Jacob Lackovic for technical assistance on this project.

G.D. and T.J.P. were supported by Alder Biopharmaceuticals. The remaining authors declare no competing financial interests.

Correspondence should be addressed to Gregory Dussor at Gregory.Dussor1@utdallas.edu. touch, as well as nausea and vomiting. Globally ranked as the second highest cause of disability (GBD 2016 Disease and Injury Incidence and Prevalence Collaborators, 2017), migraine is $2-3$ times more common in women than in men. Further, migraine is the most common cause of disability in women ages 15-49 (Steiner et al., 2018). Little is known as to why sex differences exist in migraine prevalence. In females, the incidence of migraine rises following the onset of puberty and decreases after menopause, suggesting an influence of hormones on the pathology

https://doi.org/10.1523/JNEUROSCI.0364-19.2019

Copyright $\odot 2019$ the authors 
(Stewart et al., 1994; Buse et al., 2013; Steiner et al., 2018). However, hormones may be only one of many contributing factors leading to the higher prevalence in women, and their downstream mechanisms may promote sex differences in the actions of numerous signaling pathways.

Calcitonin gene-related peptide (CGRP) has been implicated in the pathology of migraine for several decades. CGRP is elevated in venous blood, saliva, and CSF of migraine patients during an attack (Edvinsson and Goadsby, 1994; Jang et al., 2011). Attacks can be triggered in migraine patients by intravenous CGRP (Lassen et al., 2002) and can be treated by inhibitors of CGRP signaling (Tepper, 2018). Recent studies have further confirmed a role for CGRP because monoclonal antibodies recognizing the peptide or its receptor are efficacious at reducing the frequency of both episodic and chronic migraine in humans (Dodick et al., 2014a,b, 2018; Halker Singh et al., 2019). Importantly, because CGRP administration likely triggers attacks without crossing the blood-brain barrier, and because only a small fraction of therapeutic monoclonal antibodies reaches the brain, these data argue strongly for a role of peripheral CGRP in migraine (Iyengar et al., 2017). Although the exact location of peripheral CGRP action in migraine remains unclear, nociceptive signaling from the dura mater is considered a necessary event in the headache phase of attacks. CGRP has dilatory actions on the vasculature in human dura when given intravenously, including dilation restricted to the headache side during attacks (Asghar et al., 2010, 2011). Similar dilation of vessels in human dura on the headache side has also recently been shown during spontaneous migraine (Khan et al., 2019). These data suggest that CGRP in the dura mater, likely released by nociceptive nerve endings, is one key site of peptide action during migraine attacks.

Despite the differences in migraine prevalence between sexes and the clear role of CGRP in the disorder, surprisingly few studies have examined whether CGRP has sex-specific actions. While it has been shown that estrogen is capable of regulating the release of CGRP (Stucky et al., 2011; Pota et al., 2017), studies investigating the connection of CGRP to migraine have been almost entirely conducted in males. In prior studies that have administered CGRP to both males and females, none has compared responses to dural application of CGRP; this is surprising considering the role afferent nociceptive signaling from the meninges is thought to play in the pathology of the headache phase of migraine.

Here, we initially aimed to address whether dural application of CGRP caused behavioral responses in awake animals using preclinical migraine models. While our data support prior studies showing no effect of dural CGRP in males (Levy et al., 2005), we unexpectedly found a robust action of dural CGRP in females. This sexually dimorphic effect of CGRP occurs in both rats and mice and is present in both naive animals as well as after priming.

\section{Materials and Methods}

\section{Animals}

In this study, 12- to 14-week-old $\sim 260-300 \mathrm{~g}$ female and $\sim 300-350 \mathrm{~g}$ male Sprague Dawley rats (Taconic) and 6- to 8-week-old female and male ICR mice (Envigo) were used for experiments. Animals were housed on a $12 \mathrm{~h} \mathrm{light/dark} \mathrm{cycle} \mathrm{with} \mathrm{access} \mathrm{to} \mathrm{food} \mathrm{and} \mathrm{water} \mathrm{ad} \mathrm{libitum.}$ Animals were housed in the facility for at least $72 \mathrm{~h}$ before handling and habituation of animals to testing rooms. In all experiments, investigators were blinded to treatment groups. All procedures were conducted with prior approval of the Institutional Animal Care and Use Committee at the University of Texas at Dallas.

Rat cannula implantation and drug delivery

Dural injections in rats were administered via cannula at a total of $10 \mu \mathrm{l}$ injections of CGRP, interleukin-6 (IL-6), or synthetic interstitial fluid
(SIF) as vehicle (see Table 1). Animals were anesthetized initially at 5\% isoflurane via a nose cone; once animals no longer demonstrated a paw pinch reflex, isoflurane was lowered to $2.5 \%-3.5 \%$ for the entirety of the surgery. The scalp was incised longitudinally and retracted from the midline to expose the skull. Using a pin vise (Grainger Industries) set to a length of $1 \mathrm{~mm}$, a $1 \mathrm{~mm}$ burr hole was created using a stereotaxic frame at the target coordinates to sit above the middle meningeal artery $(8 \mathrm{~mm}$ anteroposterior, $-2 \mathrm{~mm}$ mediolateral, $1 \mathrm{~mm}$ dorsoventral) to puncture the skull while leaving the dura intact. A guide cannula (Plastics One C313G/SPC gauge 22) was implanted into the burr hole using a stereotaxic frame and sealed using Vetbond (3M). Two screws were inserted above the guide cannula on both sides of midline below bregma. Perm reline repair resin (Coltene) was used to anchor the cannula to the screws and skull. To prevent clogging, a dummy cannula (Plastics One 313DCSPC $0.014-0.36 \mathrm{~mm}$ fit $1 \mathrm{~mm}$ ) was inserted into the guide cannula. After surgery, animals were given $8 \mathrm{mg} / \mathrm{kg}$ gentamicin and $0.25 \mathrm{mg}$ meloxicam to prevent infection and for pain management, respectively. Animals were returned to their home cage and allowed to recover for $7 \mathrm{~d}$.

\section{Mouse dural injections}

Mouse dural injections were performed as previously described (BurgosVega et al., 2019). Mice were anesthetized under isoflurane for $<2 \mathrm{~min}$ $<2.5 \%-3 \%$ isoflurane via a nose cone. While anesthetized, treatments were injected in a volume of $5 \mu \mathrm{l}$ via a modified internal-cannula (Invivo1, part \#8IC313ISPCXC, Internal Cannula, standard, 28 gauge, fit to $0.5 \mathrm{~mm}$ ). The inner projection of the cannula was used to inject through the soft tissue at the intersection of the lambdoidal and sagittal sutures. The length of the projection was adjusted, using calipers, to be from 0.5 to $0.65 \mathrm{~mm}$ based on the animal weight $(30-35 \mathrm{~g})$ as to not puncture the dura.

\section{Rat intracisternal injections}

Intracisternal injections were administered in a volume of $10 \mu \mathrm{l}$ at a rate of $1 \mu \mathrm{l} / \mathrm{s}$ and performed in rats as previously described (Chen et al., 2013). A 25 gauge, 1.5 inch needle was contorted $\sim 7 \mathrm{~mm}$ from the tip at a $45^{\circ}$ angle with the bevel facing outwards. The needle was attached to a 25 gauge Hamilton syringe. Animals were anesthetized for $<2$ min $<2.5 \%-3 \%$ isoflurane via a nose cone. The head of the animal was tilted forward at an $\sim 120^{\circ}$ angle between body to allow access to the cisterna magna. The needle was positioned above $\mathrm{C} 1$ and inserted through the cisterna magna along the midline.

\section{Intraplantar injections}

Rats were anesthetized initially at $5 \%$ isoflurane via a nose cone; once animals no longer exhibited a pinch reflex, isoflurane was lowered to $2.5 \%-3 \%$, during which time animals were injected. These animals received a volume of $50 \mu \mathrm{l}$ into the left hindpaw via injection with a 30 gauge, 0.5 inch needle attached to a Hamilton syringe. Animals were kept under isoflurane for $<2 \mathrm{~min}$.

\section{von Frey testing}

Rats. Animals were conditioned for 5 continuous minutes by handling, $24 \mathrm{~h}$ before habituation. Rats were habituated to testing chambers and testing room by being placed in testing chambers for $2 \mathrm{~h} / \mathrm{d}$ for $3 \mathrm{~d}$ before the first day of testing. Food was placed in the chamber of each rat for the duration of the testing day. Mechanical sensitivity baselines were assessed before drug administration. Only animals that met a facial baseline threshold of $8 \mathrm{~g}$ (rats) or $0.6 \mathrm{~g}$ (mice) were included in the study. These animals were then randomly allocated to experimental groups and remained in the study until completion of the experiment. Immediately after baseline, animals were injected; and $1 \mathrm{~h}$ after injection, mechanical sensitivity thresholds were assessed both on the periorbital region and hindpaw for a maximum of $5 \mathrm{~s}$, or shorter if the investigator observed a response. von Frey filament thresholds were determined by the Dixon "up-and-down" method. Testing began with the $1 \mathrm{~g}$ filament on the face and $2 \mathrm{~g}$ on the hindpaw and increased weight to a maximum of $8 \mathrm{~g}$ on the face and $15 \mathrm{~g}$ on the hindpaw. For acute pain, animals were tested every other hour until a maximum of $5 \mathrm{~h}$ after injection was reached. Animals were then tested once every day from the time of injection until animals returned to baseline. Once animals returned to baseline, a normally sub- 
Table 1. Drugs administered in these experiments, sources, doses/volumes, and routes of administration

\begin{tabular}{|c|c|c|c|}
\hline Drug & Source & Dose & Administration route \\
\hline$\alpha$-Rat CGRP & Bachem & $\begin{array}{r}3.8 \mu \mathrm{g} / 10 \mu \mathrm{l} ; 3.8 \mathrm{ng} / 10 \mu \mathrm{l} \\
1 \mathrm{pg} / 10 \mu \mathrm{l} ; 0.1 \mathrm{pg} / 10 \mu \mathrm{l}\end{array}$ & Dural; intraplantar \\
\hline Rat recombinant IL-6 & R\&D Systems & $0.1 \mathrm{ng} / 10 \mu \mathrm{l}$ & Dural \\
\hline $\begin{array}{l}\text { Human recombinant } \\
\text { BDNF }\end{array}$ & R\&D Systems & $1 \mathrm{pg} / 10 \mu \mathrm{l}$ & Intracisternal \\
\hline SNP & Sigma-Aldrich & $0.1 \mathrm{mg} / \mathrm{kg}$ & Intraperitoneal \\
\hline
\end{tabular}

threshold stimulus was administered to examine the ability of the initial stimulus to cause priming. The same testing times were maintained following priming stimuli. All investigators were blinded to experimental conditions.

Mice. Mice were conditioned for 5 continuous minutes by handling, $24 \mathrm{~h}$ before habituation. Mice were habituated to paper cups (Choice $4 \mathrm{oz}$ paper cups: $6.5 \mathrm{~cm}$ top diameter, $4.5 \mathrm{~cm}$ bottom diameter, $72.5 \mathrm{~cm}$ length) while in testing chambers (Burgos-Vega et al., 2019). Each mouse typically used their same assigned paper cup for the remainder of the experiment. Animals were given food while in testing chambers to allow for testing as previously described (Burgos-Vega et al., 2019). Filament thresholds were determined using the Dixon "up-and-down" method. Testing in mice began with $0.07 \mathrm{~g}$ on the face and increase weight to a maximum of $0.6 \mathrm{~g}$ on the face. Mice maintained the same testing timeline as rats. All investigators were blinded to experimental conditions.

\section{Grimace}

Discomfort following treatment was recorded in male and female mice in five characterized areas (orbital, nose, cheek, ears, and whiskers) on a 3 point scale $(0=$ not present; $1=$ moderate; and $2=$ obviously present $)$ as previously characterized (Langford et al., 2010). Grimace was conducted on mice before von Frey testing for all time points. All investigators were blinded to experimental conditions.

\section{Drugs}

$\alpha$-Rat CGRP (Bachem) stock solution was prepared in $\mathrm{ddH}_{2} \mathrm{O}(1 \mathrm{mg} / \mathrm{ml})$ and diluted in SIF consisting of $135 \mathrm{~mm} \mathrm{NaCl}, 5 \mathrm{~mm} \mathrm{KCl,} 10 \mathrm{~mm}$ HEPES, $2 \mathrm{mM} \mathrm{CaCl}_{2}, 10 \mathrm{~mm}$ glucose, $1 \mathrm{mM} \mathrm{MgCl}_{2}$ (pH 7.4, $310 \mathrm{mOsm}$ ). Rat recombinant IL-6 (R\&D Systems) stock solution $(10 \mu \mathrm{g} / \mathrm{ml})$ was prepared in sterile $0.1 \%$ BSA and diluted to $1 \mathrm{ng} / \mathrm{ml}$ in SIF. Human recombinant BDNF (R\&D Systems) stock solution was made in sterile PBS containing $1 \%$ BSA. BDNF was dissolved into aCSF containing $126 \mathrm{~mm}$ $\mathrm{NaCl}, 2 \mathrm{~mm} \mathrm{KCl}, 1.25 \mathrm{~mm} \mathrm{KH}_{2} \mathrm{PO}_{4}, 2 \mathrm{mM} \mathrm{CaCl}_{2}, 2 \mathrm{~mm} \mathrm{MgSO}_{4}$, and 11 mM glucose. Sodium nitroprusside (SNP; Sigma-Aldrich) was dissolved in sterile PBS. Details on drugs used in these studies can be found in Table 1.

\section{Experimental design and statistical analysis}

Data are presented as mean \pm SEM. Data were analyzed among groups at each time point via two-way ANOVA and followed by Bonferroni post test when appropriate. Data analysis was performed using Prism (GraphPad Software). Significance was set at $p<0.05$ for all analyses. Power analysis was performed using $G$ power for comparison of the means between groups using expected effect sizes based on pilot studies as well as previously published results in the case of dural IL-6 and cisternal BDNF (Burgos-Vega et al., 2016). For dural CGRP experiments in mice, the sample size was determined based on pilot studies with rats. In the case of CGRP administered in the hindpaw of animals, we were unsure of the effect size, so a minimum sample size of 9 was used. All sample sizes reflect or surpass the suggested sample sizes for all experiments, with a minimum of 6 animals in each group for rat experiments and a minimum of 4 for mice experiments.

\section{Results}

Dural CGRP induces mechanical sensitivity in female, but not male, rodents

Previous studies demonstrated that $100 \mu \mathrm{M}$ CGRP applied to the dura does not sensitize or excite meningeal nociceptors in anes- thetized rats (Levy et al., 2005). One important caveat to this study is that only males were used to record nociceptor activity. Because migraine is more common in females, we set out to establish whether CGRP could trigger migraine-like pain in female rats using the same concentration of CGRP from prior studies, $100 \mu \mathrm{M}$. Dural application of $100 \mu \mathrm{M}(3.8 \mu \mathrm{g})$ CGRP elicited significantly lower facial withdrawal thresholds at 3, 5, and $24 \mathrm{~h}$ after injection in females, but not males (Fig. 1A). The vehicle administration had no significant effect in either male or female rats.

We have previously shown that "priming" of the dural afferent system with IL-6 can induce sensitivity to subthreshold stimulation with decreased $\mathrm{pH}$ (7.0) after the animals returned to baseline (Burgos-Vega et al., 2016). Here we show that, $5 \mathrm{~d}$ after injection of CGRP, all animals returned to baseline withdrawal threshold. Rats were then injected with $10 \mu$ l of SIF at pH 7.0 onto the dura. Three hours following injection of $\mathrm{pH}$ 7.0 SIF, only females presented with significant allodynia, and this effect persisted for at least $24 \mathrm{~h}$ (Fig. $1 \mathrm{~A}$ ).

While female rats responded to $3.8 \mu \mathrm{g}$ CGRP, it was important to determine whether this high dose was necessary for responses in females or whether they respond to lower doses. We thus started a series of experiments where CGRP dose was lowered 10-fold in each case. The data for doses of CGRP between 3.8 $\mu \mathrm{g}$ and $3.8 \mathrm{ng}$ are not shown. Figure $1 B$ shows the results of application of the thousand-fold lower dose of $3.8 \mathrm{ng}$ CGRP on the dura of cannulated female rats. Even at this low CGRP dose, significant decreases in withdrawal thresholds at 1, 3, 5, 24, 48, and $72 \mathrm{~h}$ after injection were observed in females. Additionally, to examine whether this lower dose of dural CGRP could also prime animals to respond to a normally non-noxious stimulus, animals were given dural SIF at $\mathrm{pH} 7.0$ after they returned to baseline withdrawal thresholds, which was $5 \mathrm{~d}$ following dural CGRP. Animals that received CGRP were primed to dural pH 7.0 as they experienced significantly reduced mechanical withdrawal thresholds 3 and $24 \mathrm{~h}$ after $\mathrm{pH} 7.0$ injection (Fig. $1 B$ ). As with the high dose in Figure $1 A$, males did not respond to any lower doses of CGRP (data not shown).

The initial use of the $3.8 \mu \mathrm{g}$ value as a dose was based on the original publication using dural application of $100 \mu \mathrm{M}$ CGRP (Levy et al., 2005). We next switched to more standard dose units to determine the threshold for responses to dural CGRP in females. Following dural administration of $1 \mathrm{pg}$, but not $0.1 \mathrm{pg}$ CGRP, female rats showed significantly lower facial mechanical thresholds at 1,3 , and $5 \mathrm{~h}$ thresholds compared with rats that were administered $\left(F_{(8,85)}=11.07, p<0.0001\right.$; Fig. 2$)$. Because $0.1 \mathrm{pg}$ CGRP failed to produce a hypersensitivity response in these animals, we used this dose as a subthreshold CGRP stimulus in subsequent experiments.

\section{Induction of female-specific priming to subthreshold dural CGRP}

We have previously reported that dural injection of IL-6 can establish sustained hyperalgesic priming (Burgos-Vega et al., 2016). We thus asked whether IL-6 (0.1 ng) can sensitize the dura to a subthreshold dose of CGRP $(0.1 \mathrm{pg})$ and whether this response is also female-specific. In both male (Fig. $3 B$ ) and female rats (Fig. 3A), IL-6 induced significant allodynia from 1 to $24 \mathrm{~h}$ after administration and animals returned to baseline at $72 \mathrm{~h}$ after injection, following which, either $0.1 \mathrm{pg}$ CGRP or vehicle SIF was applied to the dura. Females showed priming to this subthreshold dose, as they exhibited significantly reduced facial withdrawal thresholds 1 and $3 \mathrm{~h}$ after injection $\left(F_{(9,176)}=7.065, p<0.0001\right.$; 
A

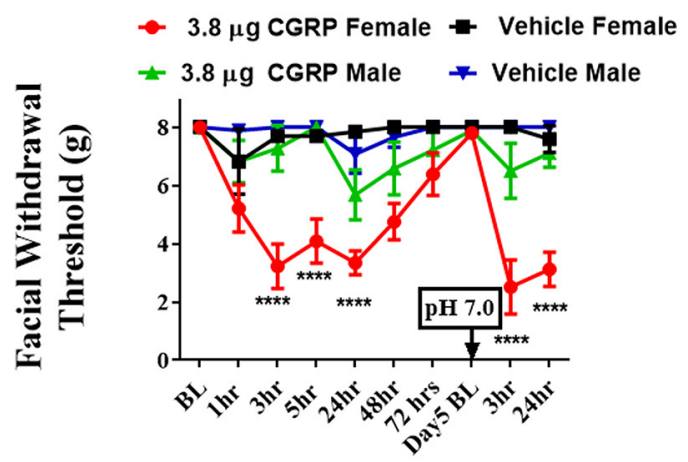

Time (h)
B

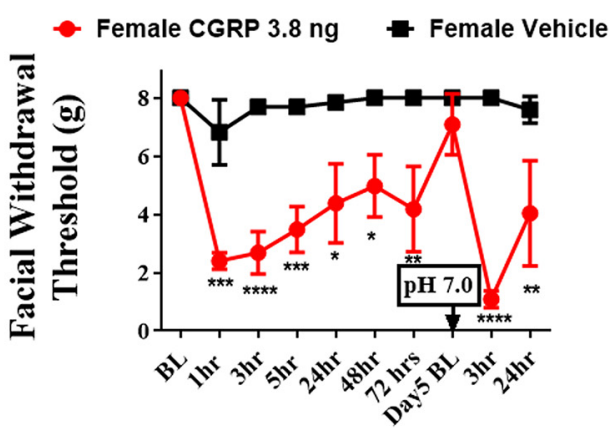

Time (h)

Figure 1. Dural administration of CGRP produces facial hypersensitivity and priming in female, but not in male, rats. Baseline facial withdrawal thresholds were determined before dural stimulation with $100 \mu \mathrm{M} \operatorname{CGRP}(\boldsymbol{A})(n=10$ females, $n=7$ males), $0.1 \mu \mathrm{M}$ CGRP ( $n=4$ females) (B), or vehicle ( $n=5$ females, $n=7$ males). A dural injection of pH $7.0 \mathrm{SIF}$ was given in all groups at day 5 following either dural CGRP or dural vehicle. Two-way ANOVA followed by Bonferroni post hoc test indicated significant differences in females treated with $100 \mu \mathrm{M} C \mathrm{CRRP}\left(F_{(27,250)}=2.591\right.$, $p<0.0001)$ and in females treated with $0.1 \mu \mathrm{M} \operatorname{CGRP}\left(F_{(27,250)}=2.591,{ }^{* * * *} p<0.0001\right)$. These data are represented as means \pm SEM. ${ }^{*} p<.05,{ }^{* *} p<.01,{ }^{* * *} p<.001$.

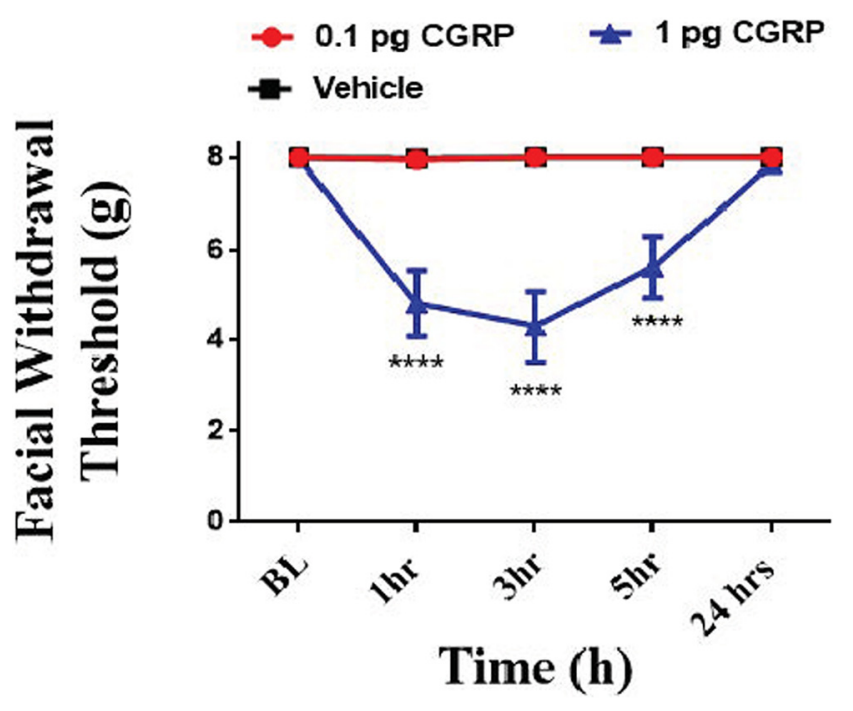

Figure2. Dural application of CGRP produces facial hypersensitivity in female rats at doses as low as $1 \mathrm{pg}$. Facial withdrawal thresholds were measured before and following dural stimulation with $0.1 \mathrm{pg}$ CGRP $(n=6), 1 \mathrm{pg}$ CGRP $(n=6)$, or vehicle pH 7.4 SIF ( $n=8)$. Two-way ANOVA followed by Bonferroni post hoc test revealed significant differences in the $1 \mathrm{pg}$ CGRPtreated group $\left(F_{(8,85)}=11.07 ;{ }^{* * * *} p<0.0001\right)$. These data are represented as means \pm SEM.

Fig. 3A). Males experienced no hypersensitivity to dural CGRP at any time point despite their initial response to IL-6 (Fig. 3B).

To determine whether initial dural stimulation was required to produce priming to subthreshold CGRP, rats were given $1 \mathrm{pg}$ intracisternal BDNF, a stimulus that we have shown previously leads to priming to dural $\mathrm{pH} 7.0$ and a systemic $\mathrm{NO}$ donor (Burgos-Vega et al., 2016). Following intracisternal BDNF, both male and female rats displayed significant facial and allodynia out to $24 \mathrm{~h}$. Once these animals returned to baseline withdrawal thresholds, at $72 \mathrm{~h}$ after injection, they were given either subthreshold $0.1 \mathrm{pg}$ dural CGRP or vehicle. Females exhibited significant facial allodynia $1 \mathrm{~h}$ after CGRP injection compared with controls $\left(F_{(9,100)}=7.467, p<0.0001\right.$; Fig. $\left.4 A\right)$. Males did not respond to CGRP at any time point following dural injection (Fig. 4B). These data demonstrate that, like dural IL-6, males respond to intracisternal BDNF but they do not prime to dural CGRP. Together, these experiments show that only females re- spond to suprathreshold CGRP doses and only females prime to CGRP following dural IL-6 or intracisternal BDNF.

\section{Female-specific responses to dural CGRP in mice}

To determine whether sex-dependent hypersensitivity following dural CGRP is a rat-specific effect, we administered CGRP to the dura of both male and female mice. Similar to what was seen with rats, female mice exhibited a reduced mechanical threshold following 1 pg dural CGRP $\left(F_{(20,121)}=4.466, p<0.0001\right)$. Female mice that received $1 \mathrm{pg}$ dural CGRP exhibited persistent facial hypersensitivity that did not return to baseline until $\sim 14 \mathrm{~d}$ after injection (Fig. 5A), much longer than rat behavior observed in Figure 1. Additionally, male mice also exhibited a marked hypersensitivity response from vehicle injection, but only at $1 \mathrm{~h}$ after injection. By $3 \mathrm{~h}$ following injection, male mice were no longer hypersensitive (Fig. 5A).

It has been shown recently that systemic administration of CGRP causes facial grimace responses suggestive of spontaneous pain (Rea et al., 2018). To examine whether the hypersensitivity responses following dural CGRP in mice are also accompanied by spontaneous pain behavior, we quantified facial grimace responses using the mouse grimace scale. Female mice that received dural CGRP had significant grimace scores out to $24 \mathrm{~h}$ after injection $\left(F_{(16,99)}=2.033, p=0.0178\right)$, whereas males that received dural CGRP or vehicle did not show grimace responses at any time point (Fig. $5 \mathrm{~B}$ ). These data show that, although male mice respond to dural CGRP, their response is minimal compared with the prolonged hypersensitivity seen in females. Additionally, dural CGRP produces behavioral responses suggestive of spontaneous pain, similar to that seen previously with systemic CGRP.

\section{Dural CGRP primes females to subthreshold SNP}

Acute administration of nitric oxide donors has been previously shown to induce migraine in humans and produce migraine-like behaviors in rodents (Olesen et al., 1993; Olesen and JansenOlesen, 2000). It has also been shown that nitric oxide applied to the dura causes CGRP release (Strecker et al., 2002). We have also shown previously that dural IL-6 or intracisternal BDNF primes rats to subthreshold doses of NO donors (Burgos-Vega et al., 2016). We next determined whether dural CGRP could prime rats to a subthreshold systemic dose of the NO donor SNP. Prior 
A

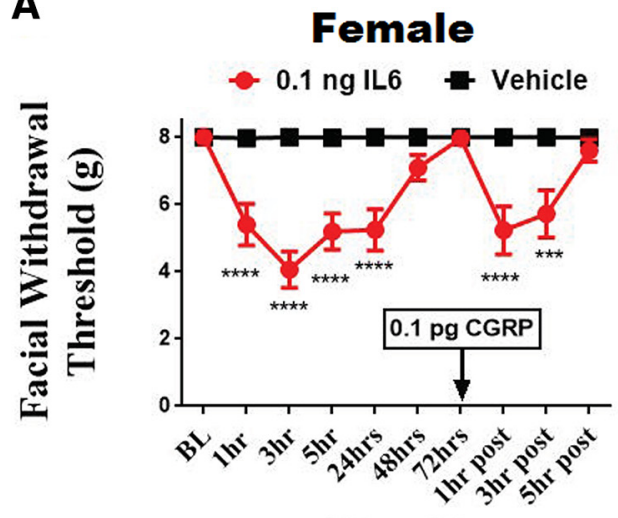

Time (h)
B

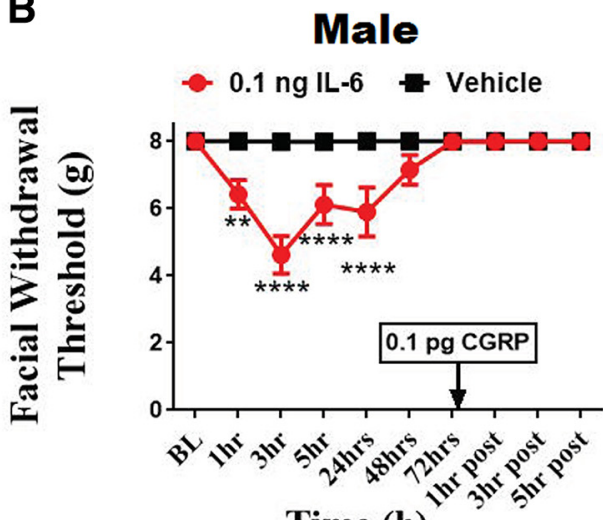

Time (h)

Figure 3. Dural application of IL-6 induces priming to dural CGRP in females, but not in males. Facial withdrawal thresholds were measured in female $(\boldsymbol{A})$ and male $(\boldsymbol{B})$ rats before and after administration of either $0.1 \mathrm{ng}$ dural IL-6 ( $n=10$ females, $n=11$ males) or vehicle $\mathrm{pH} 7.4$ SIF ( $n=9$ females, $n=11$ males). At $72 \mathrm{~h}$ after dural IL-6, all animals received a subthreshold dose of dural CGRP $(0.1 \mathrm{pg})$. Two-way ANOVA indicated a significant effect of CGRP in the IL-6-treated group of females. The significant differences of the means for each group were determined by ANOVA followed by Bonferroni post hoc test $\left(F_{(9,176)}=7.065 ;{ }^{* *} p<.01,{ }^{* * *} p<0.001,{ }^{* * * *} p<0.0001\right)$. These data are represented as means \pm SEM.

A

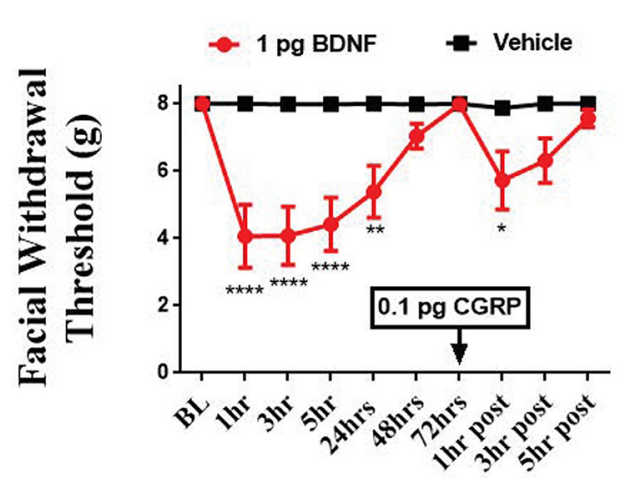

Time (h)
B Male

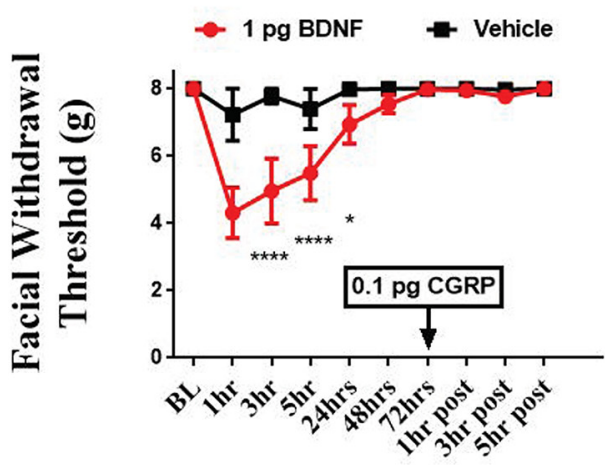

Time (h)

Figure 4. Intracisternal administration of BDNF induces priming to dural CGRP in females, but not males. Facial withdrawal thresholds were measured in female $(\boldsymbol{A})$ and male $(\boldsymbol{B})$ rats before and after either cisternal injection of $1 \mathrm{pg} \mathrm{BDNF}(n=6$ females, $n=6$ males) or vehicle (aCSF, pH 7.4) ( $n=6$ females, $n=6$ males). At $72 \mathrm{~h}$ after dural IL-6, when animals had baselined, $0.1 \mathrm{pg}$ (GRP was administered to the dura. Two-way ANOVA indicated a significant effect of CGRP in the BDNF-treated group of females. The significant differences of the means for each group were determined by ANOVA followed by Bonferroni post hoc test $\left(F_{(9,100)}=7.467\right)$. These data are represented as means \pm SEM; $\left.{ }^{*} p<.05,{ }^{* *} p<.01,{ }^{* * * *} p<0.0001\right)$.

A

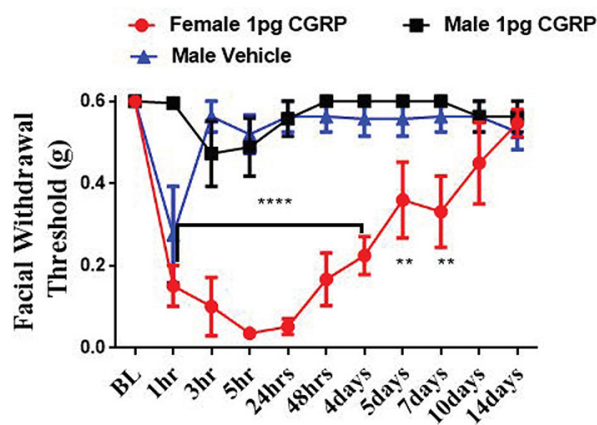

Time (h)
B

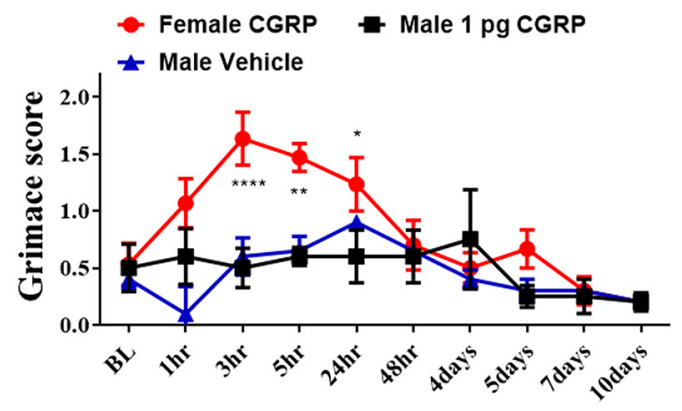

Time (h)

Figure 5. Dural application of (GRP in mice causes hypersensitivity $(\boldsymbol{A})$ and grimace $(\boldsymbol{B})$ in females, but not males. For all animals, von Frey baselines as well as Grimace Score baselines were recorded following dural injection of GRP ( $n=6$ females, $n=4$ males) or vehicle $(n=4$ males); then ANOVA with Bonferroni post hoctest showed significant differences in withdrawal thresholds female-treated group $\left(F_{(20,121)}=4.466 ;{ }^{* *} p<0.01,{ }^{* * *} p<0.0001\right)$ as well as significant grimacing $\left(F_{(16,99)}=2.033 ;{ }^{*} p<.05,{ }^{* *} p<0.01,{ }^{* * * *} p<0.0001\right)$. 


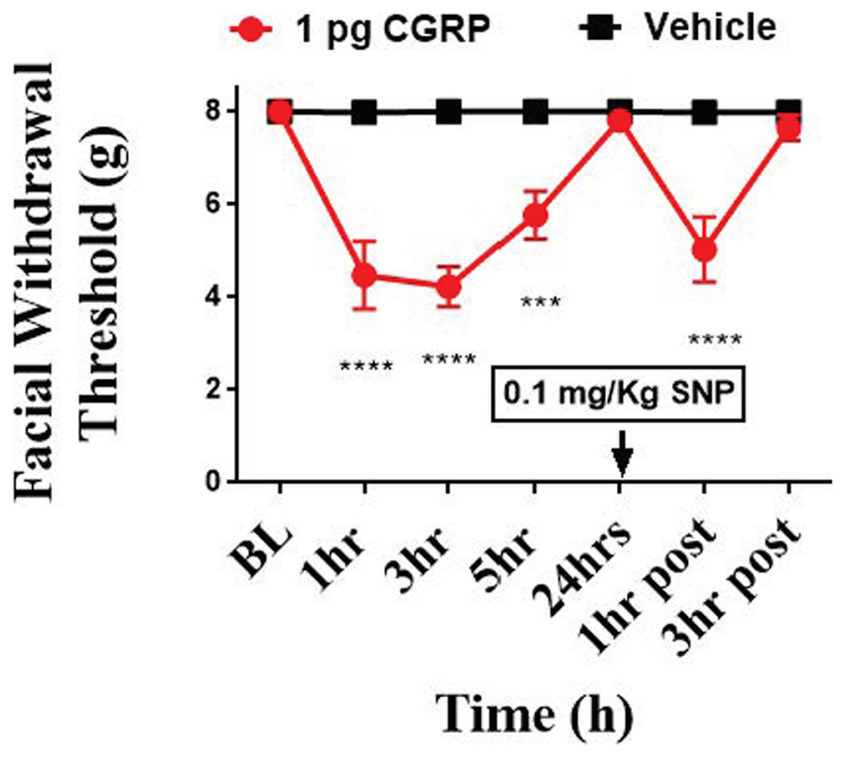

Figure 6. Dural application of (GRP primes female rats to a subthreshold nitric oxide donor. Facial withdrawal thresholds were measured in females before and after either $1 \mathrm{pg} \operatorname{CGRP}(n=$ 9) or vehicle pH 7.4SIF ( $n=7)$ applied to the dura. At $24 \mathrm{~h}$ following dural CGRP or dural vehicle, all rats were given $0.1 \mathrm{mg} / \mathrm{kg}$ SNP (i.p.). Two-way ANOVA indicated significant effects of SNP in the CGRP-treated group with no response to SNP in the vehicle-treated group. Significant differences in the means of each group were determined by ANOVA followed by Bonferroni post hoc test $\left(F_{(6,98)}=9.171\right)$. These data are represented as means \pm SEM; ${ }^{* * *} p<.001,{ }^{* * * *} p<$ $.0001)$.

studies have reported that doses up to $3 \mathrm{mg} / \mathrm{kg}$ SNP are subthreshold on otherwise naive animals (De Felice et al., 2010). Consistent with the findings in Figure 2, female rats that were administered 1 pg dural CGRP exhibited a reduction in withdrawal threshold in the facial region. Approximately $24 \mathrm{~h}$ following CGRP, female withdrawal thresholds returned to baseline (Fig. 6). Once animals returned to baseline, a dose of $0.1 \mathrm{mg} / \mathrm{kg}$ SNP was given via intraperitoneal injection. One hour after injection, females that received dural CGRP demonstrated significant facial allodynia compared with controls $\left(F_{(6,98)}=9.171, p<\right.$ 0.0001 ), showing that dural CGRP causes priming to NO donors in female rats. Because males do not show acute responses to dural CGRP, they were not tested for priming to SNP.

\section{Intraplantar CGRP produces hindpaw allodynia in female rats}

To examine whether the specificity of CGRP to induce pain in females was specific to dural administration, low-dose (1 pg) CGRP was administered into the hindpaw of otherwise naive male and female rats. At 1 and $3 \mathrm{~h}$ following injection of CGRP, females exhibited significant hindpaw allodynia compared with controls $\left(F_{(4,80)}=5.103, p=0.0010\right.$; Fig. $\left.7 D\right)$. Males that received CGRP exhibited no significant hindpaw allodynia at any time point (Fig. 7B). Neither males nor females experienced significant facial allodynia at any time point following hindpaw injections (Fig. $7 A, C$ ). This suggests that the sex specificity of pain responses following low-dose local injections of CGRP may not be specific to the dura.

\section{Discussion}

The contribution of CGRP to migraine has been investigated for $>30$ years. However, a role for peripheral CGRP has only recently been clearly demonstrated by the efficacy of predominantly peripherally restricted peptide or receptor monoclonal antibodies in human migraine patients. The location of peripheral CGRP that contributes to migraine attacks is not fully known, but given that afferent signaling from the meninges is necessary for the headache phase, and CGRP dilates meningeal vessels including on the headache side during attacks (Asghar et al., 2010, 2011), meningeal actions of CGRP are one plausible site. While the mechanisms leading to the higher prevalence of migraine in females are likely complex and not mediated by a single factor, the data shown here suggest that CGRP-based signaling from the meninges may contribute to the female-biased nature of this disorder.

Here we show that CGRP administered at doses as low as $1 \mathrm{pg}$ onto the dura of rats and mice produces facial and hindpaw allodynia in females but fails to produce allodynia in males at doses as high as $3.8 \mu \mathrm{g}$. At doses of $3.8 \mu \mathrm{g}$ and $3.8 \mathrm{ng}$, female rats were also primed to dural $\mathrm{pH}$ of 7.0 and SNP, the latter a migraine trigger in humans. This implicates dural CGRP not only in acute nociceptive responses but in the sensitization of females to normally non-noxious triggers of migraine, such as nitric oxide donors. Similar findings in 2 outbred species suggest that these femalespecific responses to dural CGRP are not a peculiarity of mice and are not an artifact of genetic manipulation, increasing confidence that these data may translate to other species. The acute responses of both males and females to dural IL-6 show that overall noxious responses to dural stimulation occur in males (see also BurgosVega et al., 2019), but that female-specific responses only occur with certain stimuli. We have also shown previously that priming to dural $\mathrm{pH} 7.0$ occurs in both males and females when the primed state is initiated by IL-6 (Burgos-Vega et al., 2016), demonstrating that priming following dural stimulation is not female-specific. Further, we have shown that the priming to dural pH 7.0 induced by intracisternal BDNF occurs in males (BurgosVega et al., 2016), and priming in the DRG/spinal dorsal horn system to BDNF also occurs in male and female rats (Moy et al., 2018). Thus, centrally induced priming is also not exclusive to females. Together, these data show that the actions of dural CGRP, and not dural signaling or priming in general, are femalespecific.

The anatomical and physiological mechanisms explaining the female-specific responses to dural CGRP are not clear. Our data showing responses to hindpaw CGRP injection only in female rats suggest that these mechanisms may be a feature of peripheral tissue and not unique to the dura. It was shown recently that CGRP administered into the peritoneal cavity does not result in differential photosensitivity and grimace between male and female mice (Mason et al., 2017), that subcutaneous CGRP given into the periorbital skin results in facial allodynia in male mice (De Logu et al., 2019), and intracisternal CGRP caused behavioral signs of headache in male rats (Cornelison et al., 2016). While these studies may seem at odds with the data shown in the current work, differential doses used in these experiments likely explain the responses in males. The most closely related study based on injection location/type is that of De Logu et al. (2019), which gave up to $15 \mathrm{nmol}$ (s.c.) under the periorbital skin, where we gave up to $3.8 \mu \mathrm{g}$ (which is $1 \mathrm{nmol}$, a dose that showed a small but nonsignificant response in males; Fig. $1 A$ ) onto the dura. In other experiments, we gave 0.1 and $1 \mathrm{pg}$ (which is 0.03 and 0.3 fmol) onto the dura. In the hindpaw experiments, we gave $1 \mathrm{pg}$, whereas other studies that found that responses in males gave intraplantar doses between 1 and $10 \mu \mathrm{g}$ (Bileviciute et al., 1998; Shi et al., 2011; White et al., 2014). Females may thus respond selectively at low doses of CGRP, whereas males respond at much 

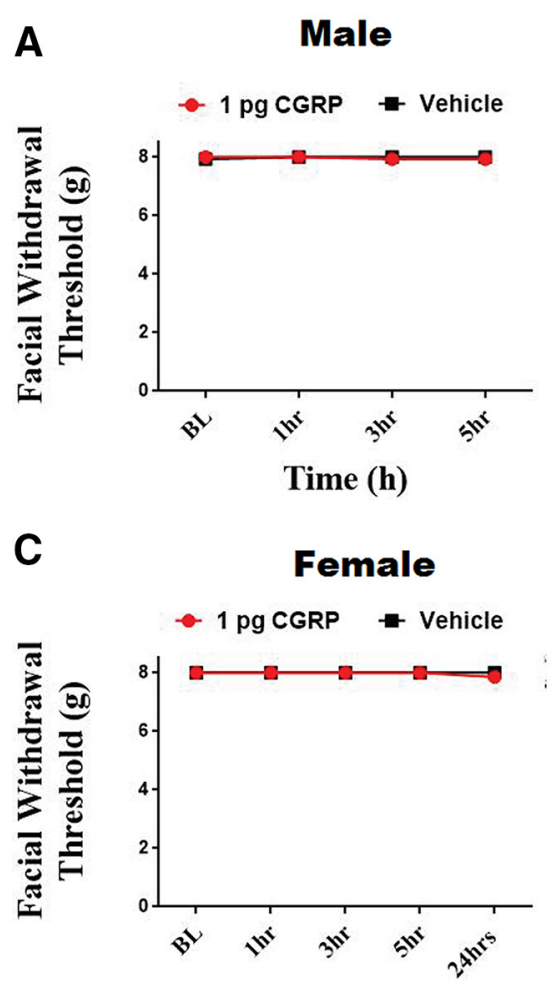

Time (h)
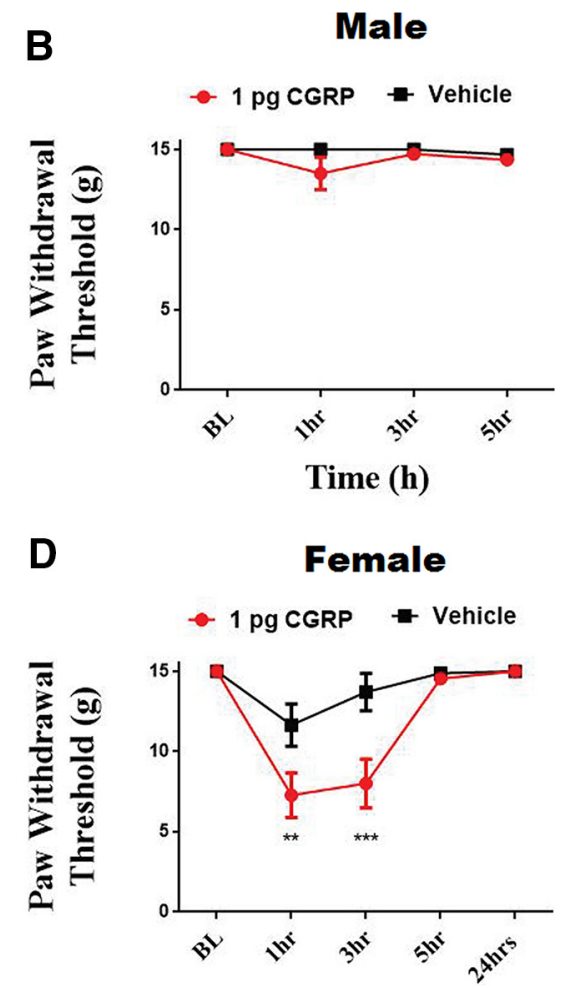

Time (h)

Figure 7. Intraplantar administration of GRP results in hypersensitivity of the hindpaw in females, but not males. Facial and paw withdrawal thresholds for males $(\boldsymbol{A}, \boldsymbol{C})$ and females $(\boldsymbol{B}, \boldsymbol{D})$ were tested before and following injection of $1 \mathrm{pg} \operatorname{CGRP}(n=9$ females, $n=10$ males) or vehicle ( $1 \times$ PBS, pH 7.4) $(n=9$ females, $n=10$ males). Two-way ANOVA followed by Bonferroni post hoc test indicated significant hindpaw allodynia in the hindpaws of CGRP-treated females $\left(F_{(4,80)}=5.103 ;{ }^{* *} p<0.01,{ }^{* * *} p<\right.$ 0.001). No significant facial hypersensitivity was observed following intraplantar (GRP administration. These data are represented as means \pm SEM to the end of this paragraph.

higher doses. Additionally, the administration route/location of CGRP may cause differential effects (i.e., systemic or into the CSF vs locally onto the dura). CGRP signaling mechanisms may only be sexually dimorphic in certain locations, and the location of action of CGRP-mediated behavioral responses was not determined in those prior studies (e.g., when given intraperitoneally or intracisternally). Nonetheless, CGRP may have sex-specific actions at nerve endings in peripheral tissue. Within the dura of rats, CGRP is expressed in C-fibers, whereas its receptor is expressed in A $\delta$ fibers; and these fibers have been reported to run in parallel with one another (Lennerz et al., 2008; Eftekhari et al., 2013). Because this study was only conducted on males, it is difficult to determine whether the presence of CGRP or the receptors would show a different expression pattern between the sexes. There have been reported differences in gene expression of CGRP as well as its receptor components. Naive female rats have significantly lower levels of RAMP1, CLR, and RCP mRNA in the trigeminal ganglion compared with male counterparts, but there is no difference in CGRP mRNA (Stucky et al., 2011). Whether or not these differences in transcript levels in the trigeminal ganglia lead to differential protein expression on nerve endings in the dura is not clear.

The female-specific actions of CGRP may be mediated in part by blood vessels where CGRP is one of the most potent endogenous vasodilators. CGRP-expressing fibers are found near both human and rat dural vessels, and CLR and RAMP1 are expressed on smooth muscle cells of these vessels (Lennerz et al., 2008). Thus, CGRP can act on smooth muscle cells, causing vasodilation as well as other dilation-independent events that may ultimately lead to afferent nociceptive signaling (Jacobs and Dussor, 2016). Unfortunately, the previously mentioned study of dural CGRP/receptor expression was conducted on male rats only, and the sample size from human tissue was too small ( 1 female and 2 males) to address sex differences in receptor expression patterns. While sex differences in the expression of CGRP receptors on blood vessels remain largely unexplored, sex-specific hormones have been shown to modulate responses within the vasculature to CGRP. When stimulated with a bolus injection of CGRP, pregnant and ovariectomized rats receiving hormone replacement of $17 \beta$-estradiol and progesterone experience larger decreases in total vascular resistance compared with ovariectomized rats not receiving hormone replacement (Gangula et al., 2001). It has also been shown that pregnant rats and hormone-treated ovariectomized rats experience larger decreases in mean arterial blood pressure in response to CGRP compared with both nonpregnant and ovariectomized rats (Gangula et al., 1999). Further, 17 $\beta$ estradiol can potentiate the dilating effects of CGRP on isolated vessels of ovariectomized rats compared with ovariectomized females with no hormone replacement (Gupta et al., 2007). These data demonstrate that female sex hormones can cause increases in vasodilation in response to CGRP.

One additional possibility explaining the sex difference in responses is that dural CGRP may cause hypersensitivity via the degranulation of mast cells native to the meninges. Degranulation of mast cells within the dura has been shown to result in excitation of meningeal nociceptors, activation of the spinal trigeminal nucleus (Levy et al., 2007), and headache behavioral responses in mice (Hassler et al., 2019). The ability of CGRP to degranulate mast cells may differ between males and females. In male rats, the CGRP receptor components CLR and RAMP1 have been located on mast cells (Eftekhari et al., 2013); however, the population of these receptor components and the potential differences in their expression on mast cells have yet to be explored in female rats. The presence of other functional CGRP receptor components, such as CTR on dural mast cells, and the possible differences in distribution of these components on mast cells are largely unexplored in both sexes. Mast cells also express receptors for estrogen as well as receptors for progesterone, both of which have been shown to degranulate mast cells (Narita et al., 2007; Jensen et al., 2010; Zierau et al., 2012). The potential interaction of estrogen and progesterone in CGRPmediated mast cell degranulation are also unexplored. In humans, it has been demonstrated that mast cell tryptase was colocalized with CLR, but not RAMP1 (Eftekhari et al., 2013). However, this finding was derived from 2 males and 1 female, too small of a sample size to be conclusive for sex differences. 
The interactions of female hormones and CGRP on mast cell degranulation requires further study before it is clear whether this mechanism contributes to the female-specific response to dural (or hindpaw) CGRP.

There are several technical limitations to the findings in this study. While cutaneous hypersensitivity is present in a large percentage of migraine patients during the headache phase of attacks (Burstein et al., 2000; Lipton et al., 2008), it is not headache. Consequently, our findings using facial von Frey testing could potentially be limited to a female-specific role of dural CGRP in the cutaneous facial hypersensitivity that is present along with migraine attacks. However, the presence of increased facial grimace responses in females, but not males, in response to dural CGRP (Fig. 5) adds an important nonevoked component to the study and demonstrates that this female-specific effect exists beyond von Frey responses. Another technical limitation of the study relates to blinding of the experimenters to the sex of the rodents. It is difficult, if not impossible, to blind those conducting behavioral responses in rodents to whether the rodent is male or female. While all other aspects of the behavioral assays are blinded, including the treatment groups, we cannot completely eliminate the impact that knowledge of the sex of the animal may have on the experimental outcomes. Multiple investigators have nonetheless observed essentially identical responses (i.e., femalespecific behaviors with dural CGRP) in experiments conducted at different times in the laboratory.

The findings shown here may raise questions regarding the efficacy of CGRP/CGRP receptor monoclonal antibodies for migraine in males. Patient demographics from published clinical trials on erenumab, fremanezumab, galcanezumab, and eptinezumab all show patient populations that are $>80 \%$ female, so it may not be clear whether these therapeutics are efficacious in a smaller percentage of males or whether higher doses may be required to achieve the same efficacy in males (Dodick et al., 2014b; Reuter et al., 2018; Rosen et al., 2018; VanderPluym et al., 2018). Alternatively, because we show that substantially lower doses of CGRP cause behavioral responses in females, the minimum concentration of CGRP necessary to contribute to migraine may be much lower in females, which may also be a contributing factor to why females are more susceptible to attacks. However, once CGRP reaches the minimum concentrations to cause effects (which again, is lower in females), the antibodies could then work equally in males and females because in either case they are simply lowering CGRP concentrations or blocking receptor signaling. Efficacy in males may also depend on the site of action of these antibodies in humans and whether they act in a location that displays sexually dimorphic CGRP signaling. If their primary site of action is not in a location where CGRP has a femalespecific effect, the antibodies will likely work equally in males and females. In any case, when considering that females make up the majority of the clinical population of migraine sufferers (Dodick et al., 2014b; Vermeersch et al., 2015; Monteith et al., 2017; Halker Singh et al., 2019), the higher percentage of females in both the clinical trials and the general patient population fortunately aligns with any female-specific efficacy of these therapeutics. While reasons for the sexually dimorphic response to dural CGRP shown here are still unknown, these findings demonstrate the need for sex-specific migraine therapeutics, as mechanisms contributing to migraine in males and females likely differ. And although CGRP plays a clear role in migraine, this does not imply that migraine is exclusively a CGRP-based disorder; other peptides, such as pituitary adenylate-cyclase activating polypeptide, may also contribute (Rubio-Beltrán et al., 2018), and there may be similar sex-specific actions of this peptide to consider. These findings also highlight the need for determining whether new therapeutics have sex-specific mechanisms of action, as selecting the proper sex in clinical trials may be key in demonstrating efficacy.

\section{References}

Asghar MS, Hansen AE, Kapijimpanga T, van der Geest RJ, van der Koning P, Larsson HB, Olesen J, Ashina M (2010) Dilation by CGRP of middle meningeal artery and reversal by sumatriptan in normal volunteers. Neurology 75:1520-1526

Asghar MS, Hansen AE, Amin FM, van der Geest RJ, Koning P, Larsson HB, Olesen J, Ashina M (2011) Evidence for a vascular factor in migraine. Ann Neurol 69:635-645.

Bileviciute I, Stenfors C, Theodorsson E, Lundeberg T (1998) Unilateral injection of calcitonin gene-related peptide (CGRP) induces bilateral oedema formation and release of CGRP-like immunoreactivity in the rat hindpaw. Br J Pharmacol 125:1304-1312.

Burgos-Vega CC, Quigley LD, Avona A, Price T, Dussor G (2016) Dural stimulation in rats causes brain-derived neurotrophic factor-dependent priming to subthreshold stimuli including a migraine trigger. Pain 157:2722-2730

Burgos-Vega CC, Quigley LD, Trevisan Dos Santos G, Yan F, Asiedu M, Jacobs B, Motina M, Safdar N, Yousuf H, Avona A, Price TJ, Dussor G (2019) Non-invasive dural stimulation in mice: a novel preclinical model of migraine. Cephalalgia 39:123-134.

Burstein R, Yarnitsky D, Goor-Aryeh I, Ransil BJ, Bajwa ZH (2000) An association between migraine and cutaneous allodynia. Ann Neurol 47: 614-624.

Buse DC, Loder EW, Gorman JA, Stewart WF, Reed ML, Fanning KM, Serrano D, Lipton RB (2013) Sex differences in the prevalence, symptoms, and associated features of migraine, probable migraine and other severe headache: results of the American Migraine Prevalence and Prevention (AMPP) study. Headache 53:1278-1299.

Chen Y, Imai H, Ito A, Saito N (2013) Novel modified method for injection into the cerebrospinal fluid via the cerebellomedullary cistern in mice. Acta Neurobiol Exp (Wars) 73:304-311.

Cornelison LE, Hawkins JL, Durham PL (2016) Elevated levels of calcitonin gene-related peptide in upper spinal cord promotes sensitization of primary trigeminal nociceptive neurons. Neuroscience 339:491-501.

De Felice M, Ossipov MH, Wang R, Lai J, Chichorro J, Meng I, Dodick DW, Vanderah TW, Dussor G, Porreca F (2010) Triptan-induced latent sensitization: a possible basis for medication overuse headache. Ann Neurol 67:325-337.

De Logu F, Landini L, Janal MN, Li Puma S, De Cesaris F, Geppetti P, Nassini R (2019) Migraine-provoking substances evoke periorbital allodynia in mice. J Headache Pain 20:18.

Dodick DW, Goadsby PJ, Spierings EL, Scherer JC, Sweeney SP, Grayzel DS (2014a) Safety and efficacy of LY2951742, a monoclonal antibody to calcitonin gene-related peptide, for the prevention of migraine: a phase 2 , randomised, double-blind, placebo-controlled study. Lancet Neurol 13: 885-892.

Dodick DW, Goadsby PJ, Silberstein SD, Lipton RB, Olesen J, Ashina M, Wilks K, Kudrow D, Kroll R, Kohrman B, Bargar R, Hirman J, Smith J (2014b) Safety and efficacy of ALD403, an antibody to calcitonin generelated peptide, for the prevention of frequent episodic migraine: a randomised, double-blind, placebo-controlled, exploratory phase 2 trial. Lancet Neurol 13:1100-1107.

Dodick DW, Silberstein SD, Bigal ME, Yeung PP, Goadsby PJ, Blankenbiller T, Grozinski-Wolff M, Yang R, Ma Y, Aycardi E (2018) Effect of fremanezumab compared with placebo for prevention of episodic migraine: a randomized clinical trial. JAMA 319:1999-2008.

Edvinsson L, Goadsby PJ (1994) Neuropeptides in migraine and cluster headache. Cephalalgia 14:320-327.

Eftekhari S, Warfvinge K, Blixt FW, Edvinsson L (2013) Differentiation of nerve fibers storing CGRP and CGRP receptors in the peripheral trigeminovascular system. J Pain 14:1289-1303.

Gangula PR, Zhao H, Supowit S, Wimalawansa S, DiPette D, Yallampalli C (1999) Pregnancy and steroid hormones enhance the vasodilation responses to CGRP in rats. Am J Physiol 276:H284-H288.

Gangula PR, Zhao H, Wimalawansa SJ, Supowit SC, DiPette DJ, Yallampalli C (2001) Pregnancy and steroid hormones enhance the systemic and 
regional hemodynamic effects of calcitonin gene-related peptide in rats. Biol Reprod 64:1776-1783.

GBD 2016 Disease and Injury Incidence and Prevalence Collaborators (2017) Global, regional, and national incidence, prevalence, and years lived with disability for 328 diseases and injuries for 195 countries, $1990-$ 2016: a systematic analysis for the global burden of disease study 2016. Lancet 390:1211-1259.

Gupta S, Villalón CM, Mehrotra S, de Vries R, Garrelds IM, Saxena PR, MaassenVanDenbrink A (2007) Female sex hormones and rat dural vasodilatation to CGRP, periarterial electrical stimulation and capsaicin. Headache 47:225-235.

Halker Singh RB, Aycardi E, Bigal ME, Loupe PS, McDonald M, Dodick DW (2019) Sustained reductions in migraine days, moderate-to-severe headache days and days with acute medication use for HFEM and CM patients taking fremanezumab: post-hoc analyses from phase 2 trials. Cephalalgia 39:52-60.

Hassler SN, Ahmad FB, Burgos-Vega CC, Boitano S, Vagner J, Price TJ, Dussor G (2019) Protease activated receptor 2 (PAR2) activation causes migraine-like pain behaviors in mice. Cephalalgia 39:111-122.

Iyengar S, Ossipov MH, Johnson KW (2017) The role of calcitonin generelated peptide in peripheral and central pain mechanisms including migraine. Pain 158:543-559.

Jacobs B, Dussor G (2016) Neurovascular contributions to migraine: moving beyond vasodilation. Neuroscience 338:130-144.

Jang MU, Park JW, Kho HS, Chung SC, Chung JW (2011) Plasma and saliva levels of nerve growth factor and neuropeptides in chronic migraine patients. Oral Dis 17:187-193.

Jensen F, Woudwyk M, Teles A, Woidacki K, Taran F, Costa S, Malfertheiner SF, Zenclussen AC (2010) Estradiol and progesterone regulate the migration of mast cells from the periphery to the uterus and induce their maturation and degranulation. PLoS One 5:e14409.

Khan S, Amin FM, Christensen CE, Ghanizada H, Younis S, Olinger ACR, de Koning PJ, Larsson HB, Ashina M (2019) Meningeal contribution to migraine pain: a magnetic resonance angiography study. Brain 142: 93-102.

Langford DJ, Bailey AL, Chanda ML, Clarke SE, Drummond TE, Echols S, Glick S, Ingrao J, Klassen-Ross T, Lacroix-Fralish ML, Matsumiya L, Sorge RE, Sotocinal SG, Tabaka JM, Wong D, van den Maagdenberg AM, Ferrari MD, Craig KD, Mogil JS (2010) Coding of facial expressions of pain in the laboratory mouse. Nat Methods 7:447-449.

Lassen LH, Haderslev PA, Jacobsen VB, Iversen HK, Sperling B, Olesen J (2002) CGRP may play a causative role in migraine. Cephalalgia 22: $54-61$.

Lennerz JK, Rühle V, Ceppa EP, Neuhuber WL, Bunnett NW, Grady EF, Messlinger K (2008) Calcitonin receptor-like receptor (CLR), receptor activity-modifying protein 1 (RAMP1), and calcitonin gene-related peptide (CGRP) immunoreactivity in the rat trigeminovascular system: differences between peripheral and central CGRP receptor distribution. J Comp Neurol 507:1277-1299.

Levy D, Burstein R, Strassman AM (2005) Calcitonin gene-related peptide does not excite or sensitize meningeal nociceptors: implications for the pathophysiology of migraine. Ann Neurol 58:698-705.

Levy D, Burstein R, Kainz V, Jakubowski M, Strassman AM (2007) Mast cell degranulation activates a pain pathway underlying migraine headache. Pain 130:166-176.

Lipton RB, Bigal ME, Ashina S, Burstein R, Silberstein S, Reed ML, Serrano D, Stewart WF (2008) Cutaneous allodynia in the migraine population. Ann Neurol 63:148-158.

Mason BN, Kaiser EA, Kuburas A, Loomis MM, Latham JA, Garcia-Martinez LF, Russo AF (2017) Induction of migraine-like photophobic behavior in mice by both peripheral and central CGRP mechanisms. J Neurosci $37: 204-216$

Monteith D, Collins EC, Vandermeulen C, Van Hecken A, Raddad E, Scherer JC, Grayzel D, Schuetz TJ, de Hoon J (2017) Safety, tolerability, pharmacokinetics, and pharmacodynamics of the CGRP binding monoclonal antibody LY2951742 (Galcanezumab) in healthy volunteers. Front Pharmacol 8:740.

Moy JK, Kuhn JL, Szabo-Pardi TA, Pradhan G, Price TJ (2018) eIF4E phos- phorylation regulates ongoing pain, independently of inflammation, and hyperalgesic priming in the mouse CFA model. Neurobiol Pain 4:45-50.

Narita S, Goldblum RM, Watson CS, Brooks EG, Estes DM, Curran EM, Midoro-Horiuti T (2007) Environmental estrogens induce mast cell degranulation and enhance IgE-mediated release of allergic mediators. Environ Health Perspect 115:48-52.

Olesen J, Jansen-Olesen I (2000) Nitric oxide mechanisms in migraine. Pathol Biol (Paris) 48:648-657.

Olesen J, Iversen HK, Thomsen LL (1993) Nitric oxide supersensitivity: a possible molecular mechanism of migraine pain. Neuroreport 4:10271030.

Pota V, Quagliariello V, Armenia E, Aurilio C, Passavanti MB, Sansone P, Iannotti M, Catauro M, Coaccioli S, Barbarisi M, Pace MC (2017) CGRP and visceral pain: the role of sex hormones in in vitro experiment. J Cell Biochem 118:510-517.

Rea BJ, Wattiez AS, Waite JS, Castonguay WC, Schmidt CM, Fairbanks AM, Robertson BR, Brown CJ, Mason BN, Moldovan-Loomis MC, GarciaMartinez LF, Poolman P, Ledolter J, Kardon RH, Sowers LP, Russo AF (2018) Peripherally administered calcitonin gene-related peptide induces spontaneous pain in mice: implications for migraine. Pain 159:2306-2317

Reuter U, Goadsby PJ, Lanteri-Minet M, Wen S, Hours-Zesiger P, Ferrari MD, Klatt J (2018) Efficacy and tolerability of erenumab in patients with episodic migraine in whom two-to-four previous preventive treatments were unsuccessful: a randomised, double-blind, placebo-controlled, phase 3b study. Lancet 392:2280-2287.

Rosen N, Pearlman E, Ruff D, Day K, Jim Nagy A (2018) $100 \%$ response rate to galcanezumab in patients with episodic migraine: a post hoc analysis of the results from phase 3 , randomized, double-blind, placebo-controlled EVOLVE-1 and EVOLVE-2 studies. Headache 58:1347-1357.

Rubio-Beltrán E, Correnti E, Deen M, Kamm K, Kelderman T, Papetti L, Vigneri S, MaassenVanDenBrink A, Edvinsson L (2018) PACAP38 and PAC1 receptor blockade: a new target for headache? J Headache Pain 19:64.

Shi X, Wang L, Li X, Sahbaie P, Kingery WS, Clark JD (2011) Neuropeptides contribute to peripheral nociceptive sensitization by regulating interleukin-1beta production in keratinocytes. Anesth Analg 113:175183.

Steiner TJ, Stovner LJ, Vos T, Jensen R, Katsarava Z (2018) Migraine is first cause of disability in under 50s: will health politicians now take notice? J Headache Pain 19:17.

Stewart WF, Shechter A, Rasmussen BK (1994) Migraine prevalence: a review of population-based studies. Neurology 44:S17-S23.

Strecker T, Dux M, Messlinger K (2002) Nitric oxide releases calcitoningene-related peptide from rat dura mater encephali promoting increases in meningeal blood flow. J Vasc Res 39:489-496.

Stucky NL, Gregory E, Winter MK, He YY, Hamilton ES, McCarson KE, Berman NE (2011) Sex differences in behavior and expression of CGRPrelated genes in a rodent model of chronic migraine. Headache 51: $674-692$.

Tepper SJ (2018) History and review of anti-calcitonin gene-related peptide (CGRP) therapies: from translational research to treatment. Headache 58 [Suppl 3]:238-275.

VanderPluym J, Dodick DW, Lipton RB, Ma Y, Loupe PS, Bigal ME (2018) Fremanezumab for preventive treatment of migraine: functional status on headache-free days. Neurology 91:e1152-e1165.

Vermeersch S, Benschop RJ, Van Hecken A, Monteith D, Wroblewski VJ, Grayzel D, de Hoon J, Collins EC (2015) Translational pharmacodynamics of calcitonin gene-related peptide monoclonal antibody LY2951742 in a capsaicin-induced dermal blood flow model. J Pharmacol Exp Ther 354:350-357.

White S, Marquez de Prado B, Russo AF, Hammond DL (2014) Heat hyperalgesia and mechanical hypersensitivity induced by calcitonin generelated peptide in a mouse model of neurofibromatosis. PLoS One 9:e106767.

Zierau O, Zenclussen AC, Jensen F (2012) Role of female sex hormones, estradiol and progesterone, in mast cell behavior. Front Immunol 3:169. 